\section{Movement of Particles in the Air}

\author{
Zoltan Csizmazia $^{1}$ - N Ilona Polyak ${ }^{2}$ \\ University of Debrecen, Centre of Agricultural Sciences, \\ ${ }^{1}$ Faculty of Agricultural Scienses, \\ Department of Machinery, Debrecen \\ ${ }^{2}$ Institute of Agricultural Economics and Rural Development, \\ Department of Agricultural Informatics and Applied Mathematics, \\ Debrecen
}

\section{SUMMARY}

The physical characteristics of particles (seeds and fertilisers) can strongly influence their movements both in seeding and spreading machines and in the air. It is therefore essential to study these particles when constructing such machines. In this respect the size, shape, coefficient of friction and aerodynamic resistance of particles are of great importance.

Due to their irregular shape, determining the size of particles is a troublesome process. A precise description of particles has to include several sizes and can be obtained from their screen size. Many physical properties of particles are relevant during movement in the air, but the aerodynamic resistance coefficient is the most important (Hofstee et al., 1990). Two types of wheat and four types of fertiliser particles were investigated (supported by the National Scientific Foundation OTKA, T-026482). An elutriator was designed and constructed (Csizmazia et al., 2000), in which an airflow is supplied by a centrifugal fan. Air velocity was measured with a thermal sensor. Particle sizes, mass and terminal air velocity were measured. The influence of the aerodynamic resistance coefficient on the particles' motion is also discussed.

\section{INTRODUCTION}

There have been experiments in the Department of Agricultural Engineering of the Centre of Agricultural Sciences, Faculty of Agronomy of Debrecen University for years to determine the physical characteristics of fertilisers, seed-corns and materials used in agriculture and the even and accurate application of seed-corns and fertilisers. This research focused on the determination of the main physical characteristics of fertiliser particles and wheat in connection with sowing and spreading machines (Csizmazia and Polyak, 1994; Csizmazia, 1990, 1993, 1998, 2000; Polyak, 1998, 2000).

For the seeds, the critical air velocity of the grains was measured and the seeds were divided into 7-17 groups, according to their critical air velocity. In our experiment, we tried to identify the factors influencing the movement of grains in the air. Thousand seed weight and grain sizes differed significantly among the groups, formed on the basis of critical air velocity.

- The thousand seed weight, generally used in practice, is an average figure representing grains with significantly different masses.

- For the various wheat types, we measured different thousand seed weights for the same critical air velocity.
- For the various wheat types, we measured different grain sizes for the same critical air velocity.

- We can conclude that the grain's movement in the air is influenced by others factors than thousand seed weight and grain size.

For the fertilisers, the particle sizes and mass, terminal air velocity was measured. The aerodynamic resistance coefficient $k$, and the terminal velocity plotted against $m_{a}$ values were calculated.

To study the aerodynamic characteristic of single particles an elutriator was designed and constructed in which an airflow is supplied by a centrifugal fan. The air velocity was measured using a thermal sensor.

\section{THEORETICAL BASIS}

Bilanski (1971) summarizes the research on aerodynamic properties of agricultural products reported in the literature for the five-year period 1966-1971.

Three experimental approaches may be used to investigate aerodynamic properties of small particles:

- the terminal velocity of the particle in free-fall through still air may be measured;

- the particles may be suspended stationary in a vertical air stream having the appropriate velocity;

- particles of different aerodynamic characteristic may be suspended simultaneously in a vertical wind tunnel, which diverges to give a linear decrease in air velocity as a function of elevation.

The aerodynamic drag coefficient of a particle may be calculated for these three methods using the measured free-fall terminal velocity or suspension air velocity and knowing particle weight and frontal area. These methods as used are either limited to the study of single particles or to very small samples of the particles (Law and Collier, 1973).

An airborne particle moving relatively to its surrounding air experiences gravitational, buoyant, frictional and inertial forces acting on it. For a given size particle moving through air at a given relative velocity, the magnitude of the aerodynamic drag coefficient $C_{d}$ is related to the Reynolds number for that particular flow condition. For a particle traversing a vertical path $y=y(t)$ in a gravitational field, the Newtonian mechanics gives the equation of motion. 


$$
m \frac{d^{2} y}{d t^{2}}=m g \frac{\left(\rho_{s}-\rho\right)}{\rho_{s}}-k\left(\frac{d y}{d t}\right)^{2}
$$

Where: $m=$ particle mass $[\mathrm{kg}]$

$g=$ gravitational acceleration $\left[\mathrm{m} / \mathrm{s}^{2}\right]$

$\rho_{\mathrm{s}}=$ particle density $\left[\mathrm{kg} / \mathrm{m}^{3}\right]$

$\rho=$ air density $\left[\mathrm{kg} / \mathrm{m}^{3}\right]$

$k=$ aerodynamic resistance coefficient $[\mathrm{kg} / \mathrm{m}]$

For grain $\rho_{s} » \rho$. Thus, when the grain attains a terminal velocity of $v_{t}$ then becomes

$\frac{d^{2} y}{d t^{2}}=0, \quad$ and $\quad \frac{d y}{d t}=v_{t} \quad$ and equation [1]

$k=\frac{m g}{v_{t}^{2}}$

Experimental measurement of particles weight and terminal velocity relative to the surrounding air permits the calculation of the aerodynamic resistance coefficient $k$ using equation [2].

Mennel and Reece (1963) found the turbulent flow regime to exist at Reynolds numbers greater than approximately 50 for small irregular-shaped particles. For a spherical particle the use of $k$ circumvents the necessity of determining the particle projected area $A$ as measured in the plane perpendicular to the velocity vector.

The resistance coefficient $k$ is related to the aerodynamic drag coefficient $C_{d}$ as

$$
C_{d}=\frac{2 k}{A \rho}
$$

In equation [2] the relative velocity $v_{t}$ may exist either due to the particle falling through still air or by a vertically-blowing air stream suspending the particle. The latter approach is the basis for the elutriation technique and an apparatus developed in this present study for the experimental determination of the aerodynamic properties of particles.

\section{MATERIALS AND METHODS}

\section{Apparatus}

An elutriator was designed and constructed with the Technical University of Budapest, Department of Fluid Mechanics. The elutriator consists of a $865 \mathrm{~mm}$ long plexiglass vertical tube with a diameter of 100 $\mathrm{mm}$ in which an airflow is supplied by a centrifugal fan. The air velocity was regulated with the modification of the fan's rpm. The air flowed from the fan through a plenum chamber upward into the $400 \mathrm{~mm}$ long test zone of the elutriator. A stainless steel screen with bore size of $0.55 \mathrm{~mm}$ (about 45 mesh) separated the test zone from the plenum chamber and supported the grain until the test began.
Many holes were bored on the mantle of the plexiglas tube along the test zone to decrease the air velocity upwards from below. This allowed the formation of a relatively flat air velocity profile in the test section. An $11 \mathrm{~mm}$ diameter hole was bored into the plexiglass tube just above the test zone for inserting the test particles.

A recovery head was connected to the top of the test channel to collect the air-entrained particles, which moved from the tube. From the recovery head, the particles fell into a collecting box.

\section{Instrumentation}

Air velocity in the bottom of the test tube was controlled by the differential pressure, as read on an inclined tube micromanometer, which was connected to a Pitot tube. Since the air velocity decreased along the test zone, it had to be measured in the different height of the test tube. Six holes were bored along the test zone to measure the air velocity with a thermal sensor (Testo 445).

\section{Calibration}

Air velocity profiles were measured in the test section of the elutriator at vertical distance (L) of 62 , 160,265 and $365 \mathrm{~mm}$ up from the lower hole line (Figure 1). The figure shows that air velocity began to decrease only 2-3 $\mathrm{mm}$ from the walls.

Figure 1: Air velocity profiles in the test section

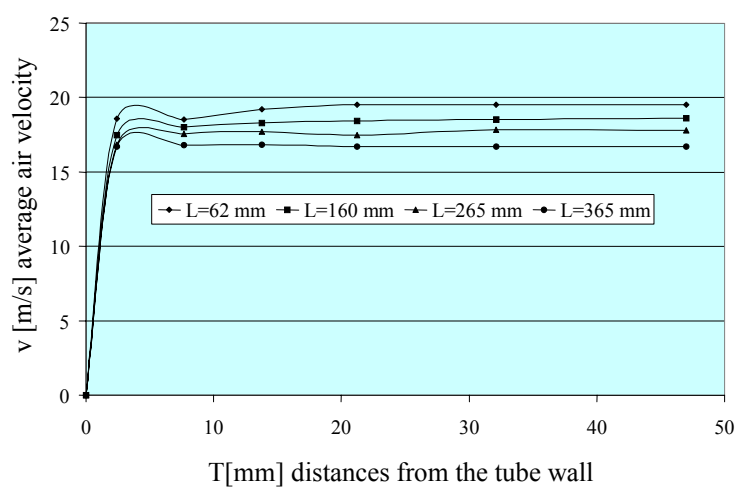

Figure 2 shows the air velocity was measured in the test section of the elutriator without the boundary layer at different vertical distances from the lower hole line. According to the Figure 2 air velocity changes approximately linearly along the axis of the test zone. The decrease of the air velocity was about $20 \%$.

Comparing the air velocity at the beginning of the test zone $\left(v_{o}\right)$ to the air velocity changing along the test zone $(v)$ gave a linear equation (Figure 3$)$.

The thermal sensor (Testo 445) was calibrated in a ventilation shaft. The results of the calibration test are seen in Figure 4. The comparison of the air velocity it can be read on the thermal sensor $\left(v_{i}\right)$ to the real air velocity $\left(v_{r}\right)$ and it gave a linear equation. 
Figure 2: Air velocity profiles in the test section

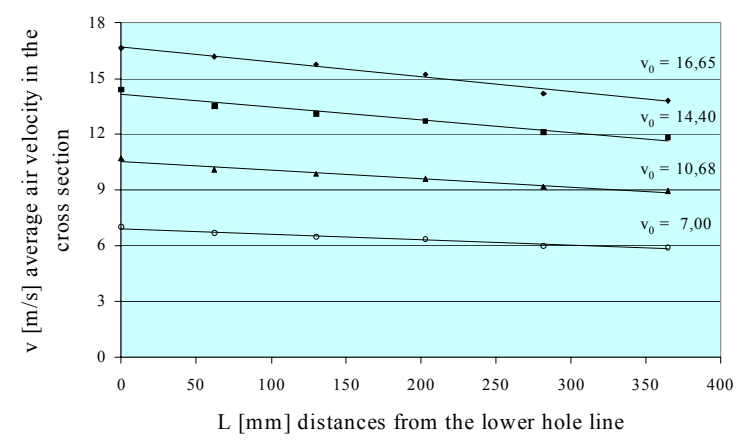

Figure 3: Velocity-changes along the test zone

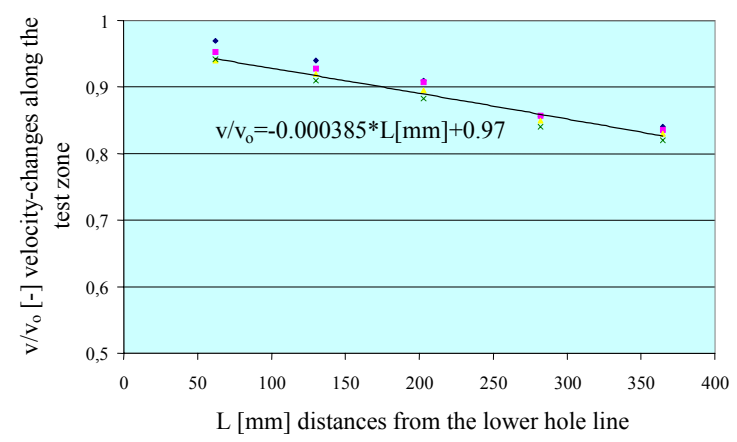

Figure 4: Calibration graph for thermal sensor

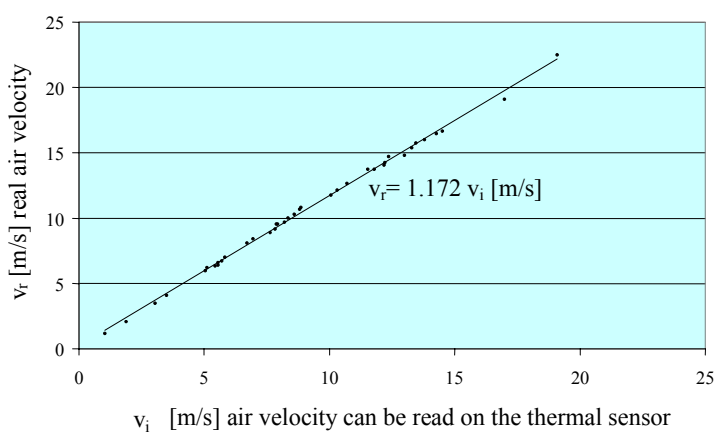

\section{Test materials}

For seeds, elutriation tests were conducted to measure the aerodynamic properties of the two different wheat types: Mv Magdalena'99 (type 1) and Kitaro trit.'99 (type 1).

Three sizes of grains (length, width, thickness) were carefully measured using a micrometer with an accuracy of $0.01 \mathrm{~mm}$, and each grain was weighed using an electronic balance capable of reading to 0.1 $\mathrm{mg}$.

For fertilisers, elutriation tests were conducted to measure the aerodynamic properties of the four different fertilisers: granular fertiliser NPK 15-15-15 (A); (obtained from tow different manufacturers $\mathrm{A}$ and B), NPK 2-18-18 (B); ammonium nitrate 34-0-0; potassium salt 0-0-60. Dimensions of particles were carefully measured along three perpendicular axes using a micrometer, and each particle was weighed using an electronic balance capable of reading to 0.1 mg.

\section{Test procedure}

For seeds, more than 200 random samples of grain were used in the test from each wheat type. From each fertiliser more than 100 random samples of particles were used in the test. A particle was placed on the stainless steel screen. The blower rpm was gradually increased until the particle was suspended in the air and the air velocity (terminal velocity) was measured with the thermal sensor. Ideal, stable conditions in the floating experiments were not obtained because particles assumed different positions within the air stream, since they rotated and tumbled due to their irregular shape and the turbulence of flow. For this reason, the average terminal velocity can be recorded when the particles were suspended in the central region of the test zone. After measurement, the particle was collected by using the blower's full speed to force it through the recovery head into the collecting box. Three replications were run in the elutriator for each grain and fertiliser.

The particle mass $m$ and the terminal air velocity $v_{t}$ were used in equation [2] to calculate the aerodynamic resistance coefficient $k$.

\section{RESULTS}

For grains, two types of wheat from the fourplaned wheat type were investigated using the above described new elutriator (Table 1).

Table 1: The main characteristics of the wheat

\begin{tabular}{|l|r|r|r|r|}
\hline & \multicolumn{2}{|c|}{ wheat type 1 } & \multicolumn{2}{c|}{ wheat type 2 } \\
\hline & $\begin{array}{c}\text { mini- } \\
\text { mum }\end{array}$ & $\begin{array}{c}\text { maxi- } \\
\text { mum }\end{array}$ & $\begin{array}{c}\text { mini- } \\
\text { mum }\end{array}$ & $\begin{array}{c}\text { maxi- } \\
\text { mum }\end{array}$ \\
\hline length $(\mathrm{mm})$ & 5,14 & 7,36 & 6,51 & 8,90 \\
\hline width $(\mathrm{mm})$ & 2,86 & 3,85 & 2,09 & 3,65 \\
\hline thickness $(\mathrm{mm})$ & 2,50 & 3,41 & 2,15 & 3,83 \\
\hline mass $(\mathrm{g})$ & 0,0278 & 0,0657 & 0,0223 & 0,0646 \\
\hline $\begin{array}{l}\text { terminal } \\
\text { velocity }(\mathrm{m} / \mathrm{s})\end{array}$ & 6,19 & 7,99 & 5,21 & 7,51 \\
\hline
\end{tabular}

Statistical investigations were done for the measured data. From the performance of an analysis of variance, it can be seen that air resistant coefficients $k_{\mathrm{type} 1}=0,009$ and $k_{\mathrm{type} 2}=0,011$ are different and characteristic of the type of wheat.

A regression analysis was also performed. The result can be seen on Figure 5 and Figure 6. The rise of the regression curve (the coefficient of $\mathrm{x}$ ) is the $\mathrm{k}$ value according to the equation [2].

The air resistant coefficients (the $\mathrm{k}$ values). $k_{\text {type } 1}=0,009$ and $k_{\text {type } 2}=0,011$. 
Figure 5: The air resistant coefficients

Wheat type 1

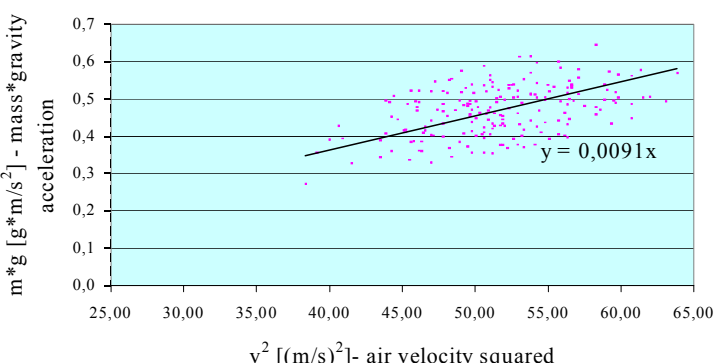

Figure 6: The air resistant coefficients

Wheat type 2

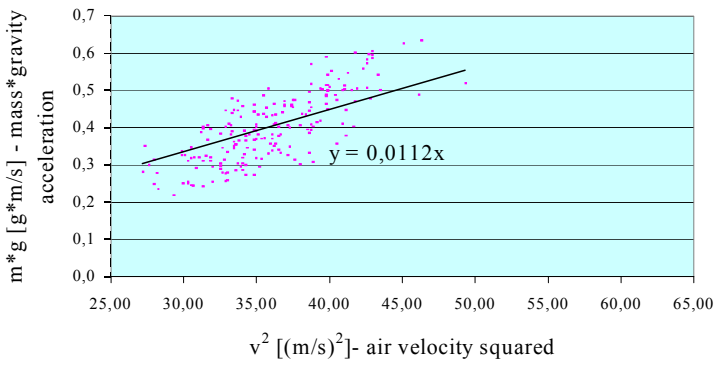

For fertiliser, 108 particles were measured from NPK 15-15-15 (A) fertiliser. Experimental values were found for particle sizes ranging from $1.81 \mathrm{~mm}$ to $8.23 \mathrm{~mm}$, particle masses ranging from $0.0069 \mathrm{~g}$ to $0.1746 \mathrm{~g}$, terminal air velocity ranging from $5.75 \mathrm{~m} / \mathrm{s}$ to $10.1 \mathrm{~m} / \mathrm{s}$. The aerodynamic resistance coefficient $k$ was calculated from the mass and terminal air velocity of the particles using equation [2] ranging from $39 \times 10^{-7} \mathrm{~kg} / \mathrm{m}$ to $55 \times 10^{-7} \mathrm{~kg} / \mathrm{m}$.

On the basis of the reasons for the experiment it can be said that the shape of the particles strongly influence the aerodynamic resistance coefficient $k$. In support of this fact a factor $m_{a}$ (the mass divided by the frontal area of the particle) was used.

$$
m_{a}=\frac{m}{A}\left[g / m^{2}\right]
$$

Figure 7 shows the terminal velocity plotted against $m_{a}$ values.

Figure 7: The ratio of terminal velocity and mass falling on a unit area

NPK 15-15-15 fertilise

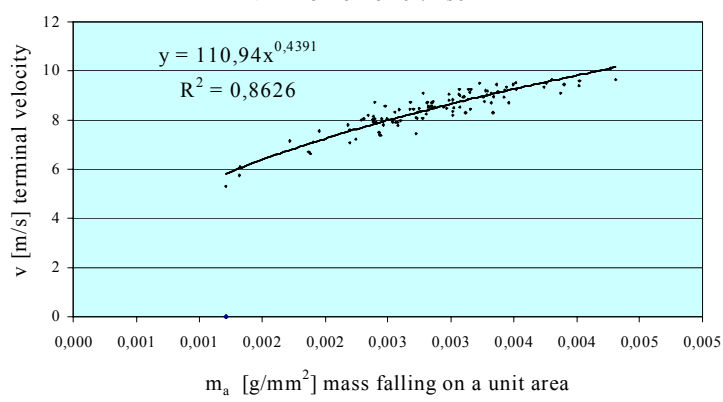

108 particles were measured from NPK 2-18-18 (B) fertiliser. Experimental values were found for particle sizes ranging from $2.45 \mathrm{~mm}$ to $15.96 \mathrm{~mm}$, particle mass ranging from $0.0139 \mathrm{~g}$ to $0.8048 \mathrm{~g}$, terminal air velocity ranging from $6.7 \mathrm{~m} / \mathrm{s}$ to 12.9 $\mathrm{m} / \mathrm{s}$, aerodynamic resistance coefficient, $k$ ranging from $13 \times 10^{-7} \mathrm{~kg} / \mathrm{m}$ to $496 \times 10^{-7} \mathrm{~kg} / \mathrm{m}$. Figure 8 shows the terminal velocity plotted against $m_{a}$ values.

Figure 8: The ratio of terminal velocity and mass falling on a unit area

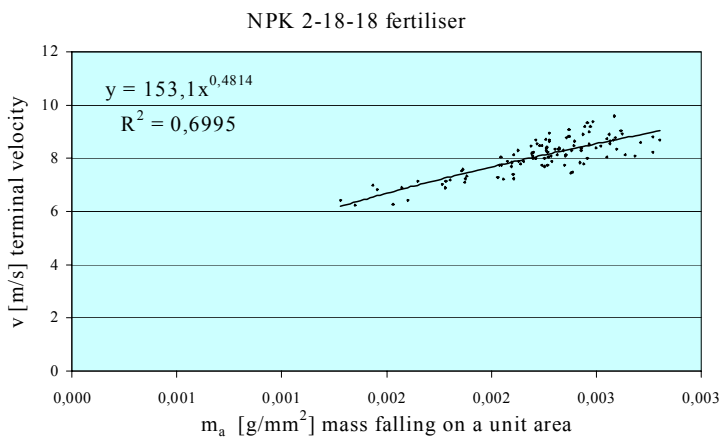

108 particles were measured from ammonium nitrate 34-0-0 fertiliser. Experimental values were found for particle sizes ranging from $1.75 \mathrm{~mm}$ to 8.22 $\mathrm{mm}$, particle mass ranging from $0.0049 \mathrm{~g}$ to 0.0772 $\mathrm{g}$, terminal air velocity ranging from $6.25 \mathrm{~m} / \mathrm{s}$ to 9.6 $\mathrm{m} / \mathrm{s}$, aerodynamic resistance coefficient, $k$ ranging from $11 \times 10^{-7} \mathrm{~kg} / \mathrm{m}$ to $179 \times 10^{-7} \mathrm{~kg} / \mathrm{m}$. Figure 9 shows the terminal velocity plotted against $m_{a}$ values.

Figure 9: The ratio of terminal velocity and mass falling on a unit area

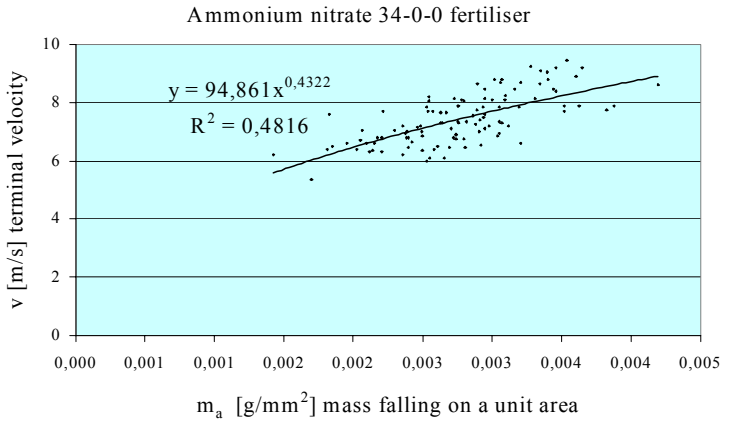

108 particles were measured from potassium salt 0-0-60 fertiliser. Experimental values were found for particle sizes ranging from $1.66 \mathrm{~mm}$ to $7.69 \mathrm{~mm}$, particle mass ranging from $0.01598 \mathrm{~g}$ to $0.1261 \mathrm{~g}$, terminal air velocity ranging from $5.35 \mathrm{~m} / \mathrm{s}$ to 9.45 $\mathrm{m} / \mathrm{s}$, aerodynamic resistance coefficient, $k$ ranging from $36 \times 10^{-7} \mathrm{~kg} / \mathrm{m}$ to $143 \times 10^{-7} \mathrm{~kg} / \mathrm{m}$. Figure 10 shows the terminal velocity plotted against $m_{a}$ values. 
Figure 10: The ratio of terminal velocity and mass falling on a unit area

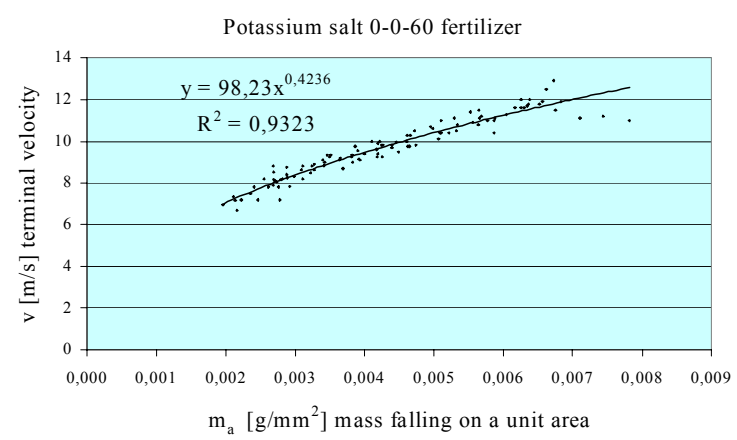

\section{DISCUSSION}

The developed elutriator could be used successfully for determining the terminal air velocity of the particles. Air velocity profiles were measured in the test section of the elutriator. The air velocity begin to decrease only $2-3 \mathrm{~mm}$ from the walls and changes approximately linear along the axis of the test zone. The decrease of the air velocity was about
$20 \%$. Comparing the air velocity at the beginning of the test zone $\left(v_{o}\right)$ to the air velocity changing along the test zone $(v)$ gave a linear equation. Comparing the air velocity can be read on the thermo sensor $\left(v_{i}\right)$ to the real air velocity $\left(v_{r}\right)$ gave a linear equation.

On the basis of the reasons for the experiment, it can be concluded that the shape of the grains strongly influence the aerodynamic resistance coefficient $k$. From the performance of an analysis of variance, it can be seen that air resistant coefficients $k_{\mathrm{type} 1}=0,009$ and $k_{\text {type } 2}=0,011$ are different and characteristic of the type of wheat. Regression analysis is also performed. The rise of the regression curve (Figure 5 and Figure 6 ) is the $k$ value.

On the basis of the reasons for the experiment, it can be said that the shape of the fertiliser particles strongly influences the aerodynamic resistance coefficient $k$. In support of this fact, a factor $m_{a}$ (the mass divided by the frontal area of the particle) was used. The $m_{a}$ value influences the terminal air velocity to a different extent for different fertilisers.

On the basis of the statistical analysis, it can be proved that the $\mathrm{R}^{2}$ values changed from 0,4816 to 0,9323 .

\section{REFERENCES}

Bilanski, W. K. (1971): Aerodynamic properties of agricultural products research, past and present. ASAE Paper, 71-846, ASAE, St. Joseph, Mich. 49085.

Csizmazia, Z. (1990): The development of fertiliser spinner for low rate fertilising. Hungarian Agricultural Engineering, 3. 22-23.

Csizmazia, Z. (1993): Technical conditions of equalised fertiliser application. Hungarian Agricultural Research, 4. 16-22.

Csizmazia, Z. (1998): Some Questions of the Development of Fertiliser Spreaders. IAMFE/ARGENTINA'98. First Latin American IAMFE Conference and Exhibition, Proceedings, 38-45.

Csizmazia, Z. (2000): Some Physical Properties of Fertiliser Particles. IAMFE/AABUK. The $11^{\text {th }}$ International Conference and Exhibition on Mechanization of Field Experiments, Proceedings, 219-226.

Csizmazia, Z.-Lajos, T.-Marshall, J.-Polyak, N. I. (2000): Új rendszerű légcsatorna fejlesztése (Developing of a New Elutriator). Mezőgazdasági Technika, 51. 9. 2-5.

Csizmazia, Z.-Polyak, N. I. (1994): The Determination of Physical
Characteristics of Seeds for the Construction of Seeding Machines. The Proceedings of the Ninth, International Conference and Exhibition on Mechanisation of Field Experiments, Beijing, China, 90-97.

Hofstee, J. W.-Huisman, W.-Speelman, L. (1990): Measuring the influence of physical properties of fertiliser on the motion of fertiliser particles. AgEng'92, Uppsala

Law, S. E.-Collier, J. A. (1973): Aerodynamic resistance coefficients of agricultural particulate determined by elutriation. Transactions of the ASAE, 5. 918-922.

Mennel, R. M.-Reece, A. R. (1963): The theory of the centrifugal distributor, III: particle trajectories. Journal of Agricultural Engineering Research, 1. 78-84.

Polyak, N. I. (1998): Some Physical Characteristics of Seeds. IAMFE-ARGENTINA'98. First Latin American IAMFE Conference and Exhibition, Proceedings, 247-255.

Polyak, N. I. (2000): Movement of Wheat Grains in the Air. IAMFE/AABUK. The $11^{\text {th }}$ International Conference and Exhibition on Mechanization of Field Experiments, Proceedings, 241-248. 